\title{
ROTULAGEM NUTRICIONAL DE PÃES INTEGRAIS: ANÁLISE E CONHECIMENTO DOS CONSUMIDORES
}

\author{
Ana Beatriz Leite, Sabrina Alves Lenquiste \\ Universidade do Oeste Paulista - UNOESTE, Curso de Nutrição, Presidente Prudente, SP.
}

\begin{abstract}
RESUMO
Avaliou-se a rotulagem nutricional e conhecimento da população sobre pães integrais. Os teores de fibras, sódio e gorduras rotulados foram avaliados. Consumidores de 3 supermercados de Presidente Prudente responderam ao questionário sobre motivos de compra e informações observadas na rotulagem de pães integrais. Todos os pães avaliados possuem no mínimo $3 \mathrm{~g} / 100 \mathrm{~g}$ de fibras, a maioria não declara a quantidade de farinha integral e todos contém o termo integral na embalagem. As faixas etárias predominantes foram 50 a 59 anos no supermercado 1 e 30 a 39 anos nos supermercados 2 e 3 . Nos supermercados 1 e 2 , predominou o sexo feminino e a observação do prazo de validade, no supermercado 3 predominou o sexo masculino e observação das calorias. Todos consumiam pela saúde e observavam a aparência. No supermercado 1 e 3 liam os rótulos as vezes e no supermercado 2 liam sempre. Faz-se necessário regulamentar os teores de sódio e gordura adicionados nesses produtos, além de estratégias de informação sobre rotulagem nutricional, possibilitando escolhas mais saudáveis e conscientes.
\end{abstract}

Palavras-chave: rótulo, pães integrais, questionário, supermercado, consumidores

\section{NUTRITION LABELING FOR INTEGRAL BREAD: ANALYSIS AND CONSUMER KNOWLEDGE}

\begin{abstract}
Nutritional labeling and knowledge of the population on whole-grain breads were evaluated. The levels of fiber, sodium and I fats labeled were evaluated. Consumers from 3 supermarkets in Presidente Prudente answered the questionnaire about purchase reasons and nutritional information of whole-grain breads. All the breads evaluated has at least $3 \mathrm{~g} / 100 \mathrm{~g}$ of fiber, most do not declare the amount of whole flour and all contain the whole-grain term in the package. The predominant age groups were 50 to 59 years in the supermarket 1 and 30 to 39 years in the supermarkets 2 and 3 . In the supermarkets 1 and 2, predominated the female sex and the observation of expiration date, in the supermarket 3 predominated males and observation of calories. Everyone consume for health and choose by appearance. In supermarket 1 and 3 they read the labels sometimes and at supermarket 2 they always read. It is necessary to regulate the levels of sodium and fat added in these products, as well as information strategies on nutrition labeling, enabling healthier and more conscious choices.
\end{abstract}

Keywords: label, whole grain bread, quiz, supermarket, consumers 


\section{INTRODUÇÃO}

Com o avanço da tecnologia, as pessoas preocupam-se mais com o trabalho e com a correria do dia-a-dia e dedicam menos tempo a alimentação, buscando formas mais fáceis e práticas para alimentar-se. Com isto o padrão alimentar vem sofrendo modificações ao longo dos anos ${ }^{1}$.

Sabe-se que os alimentos industrializados podem apresentar elevado teor de sódio, gordura, açúcar e calorias em sua constituição, além de baixo teor de fibras, sendo que esse perfil de nutrientes causa um grande impacto na saúde das pessoas ${ }^{4,5}$. 0 impacto gerado é o aumento da incidência e prevalência de doenças crônicas não transmissíveis (DCNT) como diabetes, hipertensão arterial, hipercolesterolemia, e também a aterosclerose, infarto do miocárdio e a obesidade. Em consequência há um aumento na mortalidade da população acometida por tais agravos à saúde ${ }^{6}$.

O consumo regular e adequado de fibras dietéticas é uma estratégia nutricional de grande importância na prevenção das DCNT, considerando-se como consumo adequado cerca de 20 a $35 \mathrm{~g}$ por dia para adultos e $5 \mathrm{~g}$ para crianças ${ }^{7}$.

Sabendo-se da relação do baixo consumo de fibras e alto consumo de alimentos industrializados, ricos em sódio e gorduras, com as DCNT, é necessário que haja uma mudança no perfil alimentar ${ }^{2}$. Diante disso, é de suma importância que os consumidores conheçam e entendam a rotulagem nutricional ${ }^{8}$, para que assim possam realizar escolhas mais saudáveis quando se trata de alimentos industrializados.

O rótulo é usado para informar aos consumidores quais são as propriedades nutricionais presentes nos alimentos, informando os nutrientes e o valor calórico. É uma forma de comunicação entre o fabricante e o consumidor que quer saber mais sobre o produto comprado e ter garantia de segurança ${ }^{9,10}$. Com isto, um consumidor bem informado pode fazer escolhas mais saudáveis e consequentemente prevenir doenças ${ }^{9}$.

Dessa forma, é necessário conhecer os motivos que levam os consumidores a comprarem um determinado alimento e se eles observam os rótulos, a fim de se traçar estratégias de educação nutricional para a população. Com isto, este estudo teve como objetivo avaliar o conhecimento da população sobre a rotulagem nutricional de pães integrais, bem como avaliar as informações contidas nos rótulos desses alimentos.

\section{METODOLOGIA}

Para análise das informações contidas na rotulagem nutricional dos pães integrais, foram escolhidas as marcas comercializadas em três supermercados da região central de Presidente Prudente - SP. Foram analisados os teores de gordura, de sódio e de fibras descritos nos rótulos. Os teores desses nutrientes foram comparados entre as marcas e com o referenciado pela legislação vigente para o alimento em questão.

Foi realizado ainda um estudo descritivo e de natureza transversal com os consumidores de pães integrais destes mesmos três supermercados. Os critérios de inclusão dos sujeitos a serem entrevistados foram: ter entre 20 e 70 anos, ser alfabetizado, ser consumidor de pão integral, assinar o termo de livre esclarecimento e ter respondido corretamente o questionário. A pesquisa foi aprovada pelo Comitê de Ética em Pesquisa (CEP) da UNOESTE (Protocolo de aprovação 3677).

Os entrevistados foram abordados no momento da compra e convidados a responder 0 questionário, mediante a assinatura do Termo de Consentimento Livre e Esclarecido. 0 questionário semiestruturado consistiu em seis questões de múltipla escolha, que englobaram a idade, o sexo, os motivos que levam os consumidores a comerem pão integral, o que eles consideram no momento da compra, se possuem o hábito de leitura dos rótulos e o que eles observam no rótulo dos pães integrais. Os entrevistados podiam assinalar mais de um item nas 
questões: os motivos que levam os consumidores a comerem pão integral, o que eles consideram no momento da compra e o que eles observam no rótulo dos pães integrais.

\section{RESULTADOS}

Tabela 1. Descrição e teores de gordura, sódio e fibras dos pães integrais avaliados

\begin{tabular}{|c|c|c|c|c|}
\hline & Especificação & $\begin{array}{l}\text { Gorduras totais (g/ } \\
\text { porção de } 50 \mathrm{~g})\end{array}$ & $\begin{array}{c}\text { Sódio }(\mathrm{mg} / \\
\text { porção de } 50 \mathrm{~g})\end{array}$ & $\begin{array}{c}\text { Fibras (g/ porção } \\
\text { de } 50 \mathrm{~g})\end{array}$ \\
\hline \multicolumn{5}{|c|}{ Pães integrais } \\
\hline Marca 1 & Integral & 0,70 & 188 & 2,80 \\
\hline Marca 2 & Integral & 1,70 & 242 & 3,50 \\
\hline Marca 3 & $100 \%$ Integral & 1,80 & 186 & 3,80 \\
\hline Marca 4 & $\begin{array}{l}\text { 100\% Integral Girassol e } \\
\text { Castanha }\end{array}$ & 2,70 & 186 & 4,00 \\
\hline Marca 5 & $\begin{array}{l}\text { Integral Castanha do Pará e } \\
\text { Quinoa }\end{array}$ & 2,50 & 179 & 3,40 \\
\hline Marca 6 & Integral & 3,00 & 221 & 3,10 \\
\hline Marca 7 & Integral Aveia & 3,20 & 215 & 5,30 \\
\hline Marca 8 & Integral Centeio e Gergelim & 0,90 & 78 & 4,10 \\
\hline Marca 9 & Integral Quinoa & 1,10 & 73 & 3,60 \\
\hline Marca 10 & Integral Linhaça e Trigo & 0,80 & 67 & 3,60 \\
\hline Marca 11 & Integral Ômega 3 & 1,20 & 79 & 4,30 \\
\hline Marca 12 & Integral Ômega 3 & 1,50 & 89 & 3,80 \\
\hline Marca 13 & $\begin{array}{l}100 \% \text { Integral Grãos e } \\
\text { Castanhas }\end{array}$ & 2,80 & 151 & 3,40 \\
\hline Marca 14 & Integral Zero \% & 0,70 & 208 & 4,30 \\
\hline \multicolumn{5}{|c|}{ Pães Integrais acima de 7 grãos } \\
\hline Marca 1 & Integral 15 Grãos & 1,20 & 75 & 4,00 \\
\hline Marca 2 & $100 \%$ Integral 14 Grãos & 1,90 & 159 & 3,50 \\
\hline Marca 3 & Zero \% 12 Grãos & 2,30 & 190 & 4,20 \\
\hline Marca 4 & $100 \%$ Integral 12 Grãos & 2,30 & 164 & 3,60 \\
\hline Marca 5 & Integral 7 Grãos & 3,00 & 167 & 4,00 \\
\hline Marca 6 & Integral 7 Grãos & 2,60 & 183 & 2,90 \\
\hline \multicolumn{5}{|c|}{ Pães Integrais Light } \\
\hline Marca 1 & Integral Light & 1,40 & 187 & 4,70 \\
\hline Marca 2 & 7 Grãos Light & 1,30 & 159 & 4,30 \\
\hline Marca 3 & Aveia Light & 1,00 & 175 & 3,70 \\
\hline
\end{tabular}


Comparando os pães integrais (Tabela 1), observou-se grande variação nas quantidades declaradas de fibras, gorduras totais e sódio entre as marcas. Para fibras, observou-se que os pães que apresentam mais fibras, acima de $4 \mathrm{~g}$ por porção, correspondem a $43 \%$ do total analisado, de 3 a $4 \mathrm{~g}$ corresponde a $48 \%$ e abaixo de $3 \mathrm{~g}$ corresponde a $9 \%$. Quanto às gorduras totais, observou-se que $13 \%$ dos pães avaliados apresentam mais de $3 \mathrm{~g}$ de gordura por porção, de 2 a $3 \mathrm{~g}$ corresponde a $26 \%$ e até $2 \mathrm{~g}$ corresponde a $61 \%$ dos pães. Já na avaliação do teor de sódio, notou-se que $26 \%$ dos pães tinham menos que $100 \mathrm{mg}$, 57\% tinham entre 100 e $200 \mathrm{mg}$ e $17 \%$ tinham mais que 200mg de sódio por porção. A quantidade de gorduras, fibras e sódio presentes em cada marca, descritas na tabela 1, corresponde a porção referida na embalagem do pão (50g).

Do total de 23 pães integrais avaliados, 100\% estavam de acordo com a RDC № 54, de 12 de Novembro de 2012, que determina a utilização do termo fonte de fibras. A declaração de \% de farinha integral, cuja presença na rotulagem é obrigatória de acordo com a RDC no 90, de 18 de outubro de 2000 , estava presente apenas em $47,82 \%$ dos pães avaliados. Quanto ao uso de pelo menos $51 \%$ de farinha integral, sendo esta indicada como primeiro ingrediente na lista de ingredientes, de acordo com a Lei n. 5.081-A, 100\% dos pães avaliados estavam adequados à legislação.

Na tabela 3 são apresentadas as informações de perfil dos consumidores e informações que observam na rotulagem nutricional de pães integrais. 
Tabela 3. Perfil dos consumidores e informações observadas na rotulagem nutricional.

\begin{tabular}{|c|c|c|c|}
\hline & Supermercado 1 & Supermercado 2 & Supermercado 3 \\
\hline Total de entrevistados & 12 & 50 & 3 \\
\hline \multicolumn{4}{|l|}{ Sexo (\%) } \\
\hline Sexo Feminino & 83 & 87 & 33 \\
\hline Sexo Masculino & 17 & 13 & 67 \\
\hline \multicolumn{4}{|l|}{ Idade (\%) } \\
\hline $20-29$ anos & 25 & 20 & - \\
\hline $30-39$ anos & - & 27 & 67 \\
\hline $40-49$ anos & 25 & 20 & 33 \\
\hline $50-59$ anos & 42 & 20 & - \\
\hline $60-70$ anos & 8 & 13 & - \\
\hline \multicolumn{4}{|l|}{ Motivo de consumo (\%) } \\
\hline Saúde & 100 & 100 & 100 \\
\hline Estética & 25 & 20 & 33 \\
\hline Sabor & ND & ND & ND \\
\hline Indicação médica & 8 & ND & 7 \\
\hline Inidcação nutricionista & ND & 13 & 33 \\
\hline \multicolumn{4}{|c|}{ Fatores que mais afetam a escolha (\%) } \\
\hline Preço & 33 & 60 & 33 \\
\hline Aparência & 83 & 53 & 67 \\
\hline Tamanho / quantidade & ND & ND & ND \\
\hline \multicolumn{4}{|c|}{ Hábito de leitura do rótulo (\%) } \\
\hline Lê sempre & 33 & 53 & 33 \\
\hline Lê às vezes & 50 & 40 & 67 \\
\hline Não lê & 17 & 07 & 00 \\
\hline \multicolumn{4}{|c|}{ Informações que observa no rótulo (\%) } \\
\hline Prazo validade & 92 & 87 & ND \\
\hline Lista ingredientes & 75 & 67 & ND \\
\hline Calorias & 42 & ND & 100 \\
\hline Carboidratos & ND & ND & 67 \\
\hline Proteínas & ND & ND & ND \\
\hline Gordura saturada & ND & ND & ND \\
\hline Gordura insaturada & ND & ND & ND \\
\hline Gordura Trans & 42 & ND & ND \\
\hline Fibras & ND & 60 & 67 \\
\hline Sódio & ND & ND & ND \\
\hline
\end{tabular}

*ND = não declarado

\section{DISCUSSÃO}

Os alimentos integrais apresentam muitos benefícios à saúde humana, por terem em sua composição, nutrientes essenciais ao organismo, como as fibras ${ }^{11}$. A associação do consumo de fibras com a baixa ingestão de gorduras e sódio é apontada como um fator de diminuição do risco de diversas doenças metabólicas ${ }^{3}$.

De acordo com a RDC no 54 de 12 de novembro de $2012^{12}$, para que o alimento seja considerado fonte de fibras, conforme indicado na embalagem, é obrigatório que tenha no mínimo $3 \mathrm{~g}$ de fibras a cada $100 \mathrm{~g}$ do alimento. Com isto observou-se que $100 \%$ pães analisados estavam adequados a RDC. Contrapondo-se ao nosso resultado, em estudo feito por Silva, Gallon 
e Theodoro (2014) ${ }^{13}$, apenas $50 \%$ dos rótulos estavam adequados à legislação. Assim como no estudo realizado por Paulo, Silva, Fornari, Bernabe, Arçari, Ferraz (2013) ${ }^{14}$, os pães avaliados não tinham as quantidades mínimas de fibras recomendadas.

Verificou-se que somente $47,82 \%$ dos pães declararam a quantidade de farinha integral utilizada, informação obrigatória de acordo com a RDC no 90 de 18 de outubro de $200{ }^{15}$, portanto os pães não estão conforme a resolução citada. Estudos anteriores também observaram que os pães integrais não cumprem com a legislação que dispõe a necessidade de indicação do percentual de farinha integral na rotulagem ${ }^{13,16}$.

A indicação do termo "integral" na embalagem dos pães deve ocorrer apenas para os produtos que apresentarem mais de $51 \%$ de farinha integral, sendo que este ingrediente deve aparecer como primeiro ingrediente da formulação indicado na rotulagem, com base na Lei $\mathrm{n}$. o 5.081-A de $2013^{17}$. Logo, observou-se que $100 \%$ dos pães estavam de acordo com a lei. Silva, Gallon e Theodoro (2014) ${ }^{27}$, observaram em seu estudo que apenas $73,3 \%$ dos produtos continham o termo "integral" indicado na embalagem.

Observou-se que não existem normativas específicas para os teores de gorduras e sódio nos alimentos integrais, como pães. Além disso, não foram encontrados estudos que tenham avaliado o teor destes nutrientes em pães integrais. Compreende-se a necessidade de estudos que avaliem o teor de sódio e gorduras nos alimentos integrais, bem como resoluções especificas que apontem teores máximos para tais nutrientes, tendo em vista o possível impacto negativo que, quando ingeridos em excesso, tais componentes possuem. Sem tais informações o consumidor fica sujeito a pensar que o fato de ser integral por si só torna o alimento saudável.

Em relação aos questionários respondidos, foi observada a predominância de adultos jovens (20 - 49 anos), do sexo feminino. Estudos anteriores confirmam este perfil e ressaltam que as mulheres são naturalmente mais preocupadas com a saúde e ainda são as principais responsáveis pelas tarefas domésticas diárias, inclusive a ida ao supermercado ${ }^{18,19}$.

Assim como em nosso estudo, Rodrigues, Saron e Nascimento $(2014)^{20}$, relatam que o preço é um importante fator de decisão de compra dos produtos integrais, sendo que os consumidores relatam elevado custo desses alimentos.

Quanto ao hábito de leitura dos rótulos, nosso estudo corrobora com achados anteriores que mostram que nem todos os consumidores possuem o hábito de ler sempre os rótulos, ficando abaixo de $70 \%$ também em estudos anteriores ${ }^{21,22,23}$, além disso, quando realizam a leitura dos rótulos, a informação mais procurada pelos consumidores é o prazo de validade, seguido pelo teor calórico ${ }^{22,23 .}$

Assim, ressalta-se a importância de novas legislações que determinem valores de referência para sódio e gordura em alimentos integrais, bem como se estabeleçam estratégias de informação para a população sobre rotulagem nutricional, de modo que os consumidores possam fazer escolhas saudáveis e conscientes no momento da compra.

\section{REFERÊNCIAS}

1. Gomes GMB. Cirurgia bariátrica: mudanças no padrão alimentar e na qualidade de vida. [dissertação]. Florianópolis: Universidade Federal de Santa Catarina, 2007.

2. Brognoli ML. Consumo de alimentos congelados por indivíduos frequentadores de supermercados. Universidade do Extremo Sul Catarinense. 2010.

3. Coutinho J, Gentil P, Toral N. A desnutrição e obesidade no Brasil: o enfrentamento com base na agenda única da nutrição. Cad. Saúde Pública. 2008; 24(supl. 2):332-340, https://doi.org/10.1590/S0102-311X2008001400018. 
4. Zucchi ND. Alimentos ultraprocessados direcionados a crianças: disponibilidade, informação nutricional complementar e opinião de consumidores infantis. [dissertação]. Florianópolis: Universidade Federal de Santa Catarina, 2015.

5. Maciel ES, Sonati JG, Modeneze DM, Vasconcelos JS, Vilarta R. Consumo alimentar, estado nutricional e nível de atividade física em comunidade universitária brasileira. Rev Nutr 2012; 25(6):707-718, https://doi.org/10.1590/S1415-52732012000600003.

6. Campos M, Neto J. Doenças crônicas não transmissíveis: fatores de risco e repercussão na qualidade de vida. Rev Baiana de Saúde Pública. 2009; 33(4):561-581.

7. Macedo TMB, Schmourlo G, Viana KDAL. Fibra alimentar como mecanismo preventivo de doenças crônicas e distúrbios metabólicos. Rev UNI. 2012; 2(2):67-77.

8. Gonçalves NA, Cecchi PP, Vieira RM, Santos MDA, Almeida TC. Rotulagem de alimentos e consumidor. Rev Nutr Bras. 2015; 14(4).

9. Silva LP. A influência da preocupação com a saúde no uso de informações dos rótulos de alimentos. Universidade de Brasília. 2016.

10. Cavada GS, Paiva FF, Helbig E, Borges LR. Rotulagem nutricional: você sabe o que está comendo. Braz. J. Food Technol. 2012; 15(spe):84-88.

11. Paula EP, Neres S, Santini E, Filho ADR. Considerações nutricionais para adultos com hiv/aids. Rev Matogrossense de Enfermagem. 2010; 1(2):148-165.

12. RDC 54. Regulamento Técnico sobre Informação Nutricional Complementar. ANVISA. 2012.

13. Silva VCP, Gallon CW, Theodoro H. Avaliação das rotulagens e informações nutricionais dos pães integrais: fibras, sódio e adequação com a legislação vigente. Rev Demetra. 2014; 9(4): 985-1001, https://doi.org/10.12957/demetra.2014.11979

14. Paulo KEA, Silva SC, Fornari JV, Bernabe AS, Arçari DP, Ferraz RRN. Avaliação da rotulagem de barras de cereais com relação à adequada. Rev Saúde em Foco. 2013; (7):29-34.

15. RDC 90. Regulamento técnico para fixação de identidade e qualidade de pão. ANVISA. 2000.

16. Ferraz EC, Cruz MM, Freitas EC. Avaliação dos rótulos de diferentes marcas de pães integrais versus pães sem glúten comercializados no município de Vitória da Conquista - BA. Revista Baiana de Saúde Pública. 2016; 9(1):46-55.

17. LEI 5081A. Normas de comercialização de pão integral. 2013.

18. Pimentel TC, Simões GS. Percepção dos consumidores em relação às fibras alimentares e seus produtos. Rev Bras de Pesquisa em Alimentos. 2012; 3(1):11-18, https://doi.org/10.14685/rebrapa.v3i1.56

19. Magalhães BC, Cabral NAL, Castro EEC, Oliveira ATV, Gomes RS, Sampaio GC. Consumo de fibras alimentares entre indivíduos adultos em um Supermercado de São Luís, Maranhão. Rev Pesq Saúde. 2016; 17(3):137-140. 
20. Rodrigues AMS, Saron ML, Nascimento KO. Análise do comportamento e hábitos de consumo de frequentadores de um supermercado no município de Barra Mansa - RJ. Rev Verde. 2014; $9(2): 185-194$.

21. Soares DJ, Neto LGM, Silva LMR. Análise do comportamento dos consumidores com relação à compreensão e entendimento das informações dos rótulos de alimentos. Rev AGROTEC. 2016; 37(1):105-111.

22. Machado CB, Nogueira SE, Briancini TP, Tobal TM. Avaliação do hábito de leitura e entendimento dos rótulos dos alimentos: um estudo em um supermercado na cidade de Santa Fé do Sul-São Paulo. Rev Funec Científica. 2013; 1(1).

23. Marzarotto B, Alves MK. Leitura de rótulos de alimentos por frequentadores de um estabelecimento comercial. 2017; 10(2):102-108. 